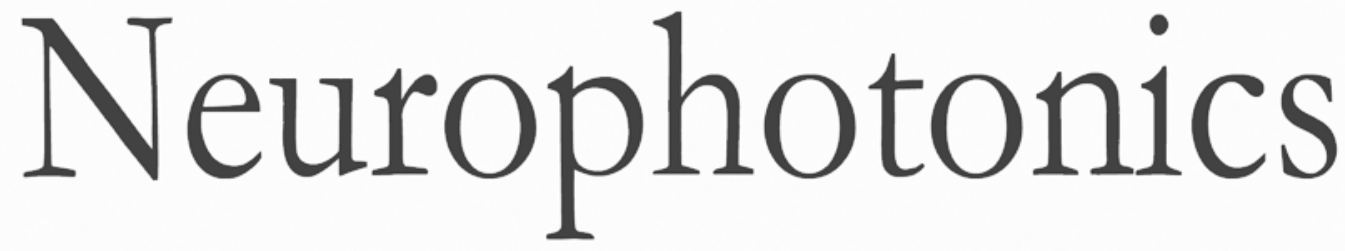

\title{
Long-term continuous monitoring of the preterm brain with diffuse optical tomography and electroencephalography: a technical note on cap manufacturing
}

\author{
Alfonso Galderisi \\ Sabrina Brigadoi \\ Simone Cutini \\ Sara Basso Moro \\ Elisabetta Lolli \\ Federica Meconi \\ Silvia Benavides-Varela \\ Eugenio Baraldi \\ Piero Amodio \\ Claudio Cobelli \\ Daniele Trevisanuto \\ Roberto Dell'Acqua
}




\title{
Long-term continuous monitoring of the preterm brain with diffuse optical tomography and electroencephalography: a technical note on cap manufacturing
}

\author{
Alfonso Galderisi, ${ }^{a, \dagger}$ Sabrina Brigadoi, ${ }^{b, *, \dagger}$ Simone Cutini, ${ }^{b, c}$ Sara Basso Moro, ${ }^{\text {d }}$ Elisabetta Lolli, ${ }^{a}$ \\ Federica Meconi, ${ }^{b}$ Silvia Benavides-Varela, ${ }^{b}$ Eugenio Baraldi, ${ }^{a}$ Piero Amodio, ${ }^{e}$ Claudio Cobelli, ${ }^{\dagger}$ \\ Daniele Trevisanuto, ${ }^{a}$ and Roberto Dell'Acqua ${ }^{b, c}$ \\ aUniversity of Padova, Neonatal Intensive Care Unit, Department of Woman's and Child's Health, via Giustiniani 3, 35128 Padova, Italy \\ bUniversity of Padova, Department of Developmental Psychology, via Venezia 8, 35131 Padova, Italy \\ 'University of Padova, Padua Neuroscience Center, via Venezia 8, 35131 Padova, Italy \\ dUniversity of Padova, Department of Neuroscience, via Giustiniani 2, 35128 Padova, Italy \\ eUniversity of Padova, Department of Medicine, via Giustiniani 2, 35128 Padova, Italy \\ fUniversity of Padova, Department of Information Engineering, via Gradenigo 6/b, 35131 Padova, Italy
}

\begin{abstract}
Diffuse optical tomography (DOT) has recently proved useful for detecting whole-brain oxygenation changes in preterm and term newborns' brains. The data recording phase in prior explorations was limited up to a maximum of a couple of hours, a time dictated by the need to minimize skin damage caused by the protracted contact with optode holders and interference with concomitant clinical/nursing procedures. In an attempt to extend the data recording phase, we developed a new custom-made cap for multimodal DOT and electroencephalography acquisitions for the neonatal population. The cap was tested on a preterm neonate (28 weeks gestation) for a 7-day continuous monitoring period. The cap was well tolerated by the neonate, who did not suffer any evident discomfort and/or skin damage. Montage and data acquisition using our cap was operated by an attending nurse with no difficulty. DOT data quality was remarkable, with an average of $92 \%$ of reliable channels, characterized by the clear presence of the heartbeat in most of them. $\odot$ The Authors. Published by SPIE under a Creative Commons Attribution 3.0 Unported License. Distribution or reproduction of this work in whole or in part requires full attribution of the original publication, including its DOI. [DOI: 10.1117/1.NPh.3.4.045009]
\end{abstract}

Keywords: diffuse optical tomography; preterm; continuous monitoring; electroencephalography.

Paper 16062TNR received Oct. 3, 2016; accepted for publication Nov. 29, 2016; published online Dec. 23, 2016.

\section{Introduction}

Functional near-infrared spectroscopy (fNIRS) is a noninvasive neuroimaging technique hinging on the properties of light in the red and near-infrared wavelength range to investigate hemodynamic activity occurring in the brain, ${ }^{1}$ enabling researchers to detect changes in oxy- $(\mathrm{HbO})$ and deoxy-hemoglobin $(\mathrm{HbR})$ concentration. When multiple sources and detectors of nearinfrared light are employed, creating multidistance channels, the technique is usually referred to as diffuse optical tomography (DOT). ${ }^{2,3}$ Depth-resolved images of $\mathrm{HbO} / \mathrm{HbR}$ concentration changes in the brain can be effectively reconstructed with DOT. $^{4}$

Optical imaging is particularly suitable for the (term and preterm) neonatal population, because of its noninvasive nature, portability - it can be used in the neonatal intensive care unit (NICU) at the cradle-side-and technical compatibility with other clinical recording systems. ${ }^{5-12}$ For instance, it can be employed in multimodal acquisitions with electroencephalography (EEG), which is used for seizure detection in clinical practice. So far, DOT studies in neonates have monitored hemodynamic changes in the whole brain up to a maximum of

*Address all correspondence to: Sabrina Brigadoi, E-mail: sabrina.brigadoi@ unipd.it

†These authors equally contributed to the work.
2 h. ${ }^{6,13-15}$ Prolonging this "monitoring window" would, however, increase the chance to document important intracranial events in the neonatal population, such as seizures or intracranial hemorrhages. ${ }^{16}$

The reasons that could explain the limited monitoring time lie primarily in (1) the discomfort of the optode holder for the baby and the skin damage that it can possibly produce and (2) a possible interference of the cap with clinical procedures and nursing. This is particularly true for DOT acquisitions, which require several fiber bundles and the placement of optodes over the whole head. This may force the newborn to a strained position and may cause skin lesions or pressure sores, especially in very preterm infants.

To this end, we propose a low-cost, easy-to-dress custommade cap with whole-head coverage for DOT and 10 electrode positions for EEG, designed for continuous DOT monitoring in the very preterm neonates. We tested the cap on a very preterm newborn in NICU with a 7-day continuous DOT monitoring in order to validate its effectiveness.

\section{Materials and Methods}

\subsection{Head Cap Manufacturing Process}

The hand-made neonatal cap (hereafter NeoCAP) was designed by merging two components: an outer layer consisting of a soft tissue cap (EasyCap, Germany) marked with the 10-10 
reference points [Fig. 1(a)] and an inner layer made of foam flexible polyurethane [Fig. 1(b)], a standard material employed for prevention of skin bedsores in the presence of prolonged forced immobilization and previously employed in preterms. ${ }^{17-19}$ Supports for optodes were created with truncated, hand-made, plastic cones, whose diameter was adapted to fit the optodes. Electrodes were inserted into white disks located in the outer layer. Holes were created in the inner layer aligned with optodes and electrodes, and the two layers were finally sewed together. With this solution, neither the plastic supports for optodes nor that for electrodes were in direct contact with the inner surface [Figs. 1(a) and 1(b)], so as to avoid the friction of the apparatus with the skin of the baby's head.

The cap was designed of a single size ( $26 \mathrm{~cm}$ circumference) corresponding to the 50th percentile of head circumference of neonates at 28 weeks gestation.

Electrodes were placed in F3, Fz, F4, C3, C4, P3, P4, and FT10 according to the international $10-5$ system. ${ }^{20}$ The ground electrode was placed on the inion and the reference electrode on FT9. Optodes were positioned in a subset of locations of the international $10-5$ system [see Fig. 1(c)].

\subsection{Validation Study}

We enrolled a neonate admitted to the NICU at the University Hospital of Padova, born at 28 weeks gestation through cesarean section for placenta abruption (birthweight: $1145 \mathrm{~g}$, in the 50th percentile, head circumference: $26.5 \mathrm{~cm}$, in the 50th percentile). Once parental consent was obtained, the NeoCAP was placed at
7 days of life and optodes were mounted. The study was approved by the Ethical Committee of the University Hospital of Padova (protocol N. 3440/AO/15).

DOT data were acquired with a multichannel continuous wave system (NIRScout, NIRx Medical Technologies LLC, Glen Head, New York), equipped with 32 LED sources (16 emitting light at $760 \mathrm{~nm}$ and 16 at $850 \mathrm{~nm}$ ) and 24 detectors. This device belongs in laser class I (eye and skin safe) and has been classified as Exempt Group according to EN 62471:2008. Only eight detectors were used in this investigation, given the small size of the head. The number of multidistance channels obtained by using the configuration shown in Fig. 1(c) was 105. The sampling frequency was set to $6.25 \mathrm{~Hz}$.

DOT data were acquired for 7 days. Interruption of the monitoring occurred four times a day for about $30 \mathrm{~min}$ at the time of routine nursing. Attending nurses easily removed and replaced the cap over the neonate's head during the procedures.

The EEG acquisition was performed for $2 \mathrm{~h}$ on day 7, simultaneously with DOT. During this time window, other than resting state, two functional auditory tasks were performed. EEG data were recorded with a portable EEG system (FirstAmp, Brain Products GmbH, Gilching, Germany) with eight channels and digitized at a sampling frequency of $250 \mathrm{~Hz}$ with a bandpass filter of 0.01 to $80 \mathrm{~Hz}$. $\mathrm{Ag} / \mathrm{AgCl}$ electrodes were used for the acquisition and electrolyte gel was introduced between the electrode and the scalp with a small syringe without needle. Impedance was kept below $20 \mathrm{k} \Omega$. The EEG signal was offline re-referenced to the average of FT9 and FT10. (a)

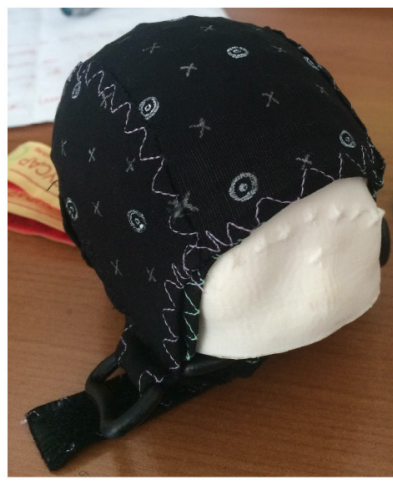

(c)

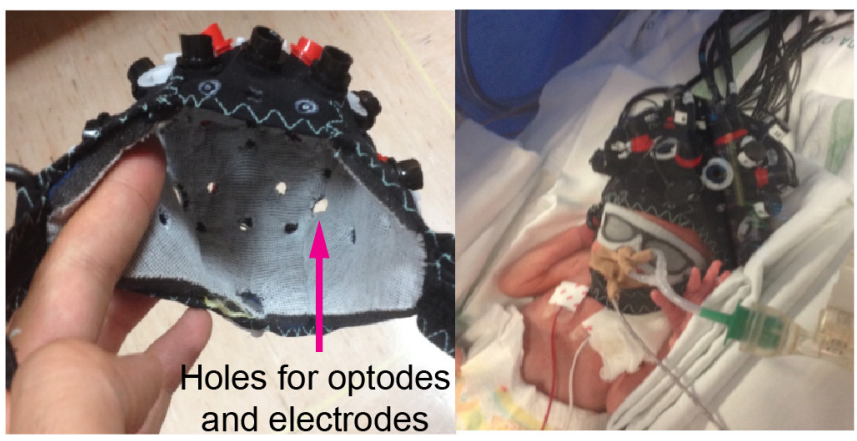

Electrode's (b)

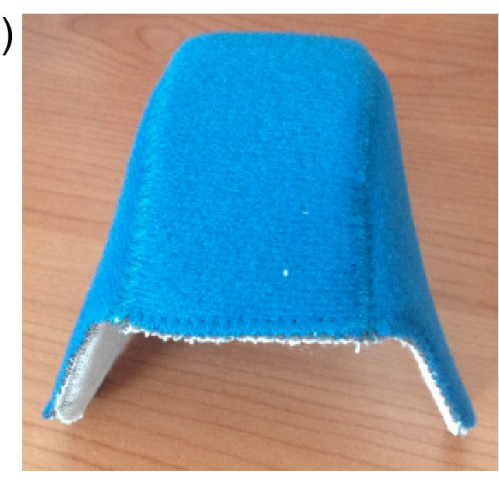

(d)

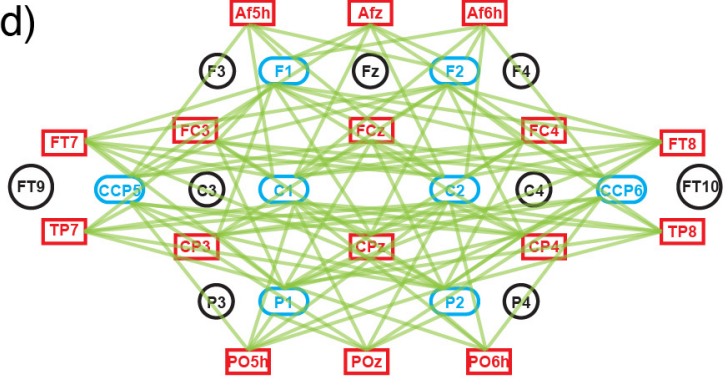

(II)

Fig. 1 (a) The original outer layer, with the 10 - 10 reference points marked on it (left) and the outer layer with all optodes' support and electrodes' support. (b) The inner layer created to match the dimension of the baby's head. (c) Outer and inner layers were sewed together to create the cap (left). The holes made in the inner layer aligned with the optodes' position and electrodes' position of the outer layer can be clearly seen. On the right, the cap positioned on the neonate's head, with all fibers connected to it. (d) Configuration of optodes and electrodes. The 10-5 locations, where optodes/electrodes were positioned, are displayed in red rectangles for sources, light blue ellipses for detectors, and black circles for electrodes. Green lines connecting sources and detectors show the 105 DOT channels. 


\subsection{Assessment}

Skin was inspected during the nursing procedures (when the NeoCAP was removed, see above) at least four times a day. Pain associated with the NeoCAP was evaluated using the premature infant pain profile (PIPP) scale $^{21}$; the assessment was performed every $6 \mathrm{~h}$.

DOT signals were partitioned in segments of different lengths. Data recorded when the cap was dislocated (identified in the signals as prolonged period of large motion artifacts or sudden drop of all signals) were removed, thus dividing the signal in several independent segments. The total time of successful monitoring was, therefore, computed as the sum of the lengths of all segments over the 7-day period.

Three metrics were used as an index of DOT data quality. First, the percentage of reliable channels for each segment was computed (measured intensity $>0.01$ and $<3$ or signalto-noise ratio $(\mathrm{SNR})>1.2$, using Homer2 fNIRS analysis toolbox ${ }^{22}$ ). Second, a time-frequency analysis was performed on DOT data at the highest wavelength to verify the presence of the heartbeat component. A spectrogram (window-length: $48 \mathrm{~s}$, hereafter time bin) was computed for each segment of data and channel. For each time bin and channel, we identified the frequency presenting the highest power spectral density in the 2- to $3-\mathrm{Hz}$ range. Then, for each time bin, the median across channels of such frequency was calculated separately for the different segments. Afterward, the mean value and the standard deviation across segments were computed as an index of average heart rate and its variability in the 7-day monitoring period. Third, a linear regression of the raw DOT signals (only reliable channels were included) was performed to rule out the presence of constant drifts contaminating the hemodynamic signal. For each segment and channel, the angle of the slope was computed as metric.

Inspection of the EEG signal acquired during functional stimulation allowed us to perform a further check on the quality of the NeoCAP by isolating segments characterized by tracé discontinu with low-voltage interburst periods, which typically flag stints of quiet sleep in preterm neonates. ${ }^{23,24}$ The presence of motion artifacts in the DOT data during these EEG-derived periods was taken as an index of the NeoCAP's fit quality and effectiveness in absence of explicit movements of the neonate.

\section{Results}

All the PIPP scale values were below 5 over the whole study period, indicating no pain associated with NeoCAP. ${ }^{21}$ No skin lesions were recorded during the observation period (Fig. 2). Accidental dislocation of optodes occurred four times over 7 days with prompt rearrangement by the attending nurse.

The neonate was successfully monitored with DOT for $122.6 \mathrm{~h}$ over the 7-day monitoring period ( $73 \%$ of the total duration).

Figure 3(a) shows a box-plot of the percentage of reliable channels for each DOT segment. Figure 3(b) shows three examples of spectrograms computed on data acquired at different days. The presence of the heartbeat component is clear as well as the variability of the frequency of the heartbeat over time, a well-established and distinctive feature in the preterm population. The mean cardiac frequency was $2.4 \pm .2 \mathrm{~Hz}$. The absence of constant drifts was attested by a mean slope angle of $.03 \mathrm{deg} \pm .1 \mathrm{deg}$, which can be considered a negligible deviation from the expected 0 deg.

Figures 4 and 5 show an example of DOT signals of all channels in one of the segments of data and an example of EEG data, respectively.

Approximately 17 min were identified in the EEG trace as featuring the tracé discontinu. During this time period, nine motion artifacts were identified in the DOT data, with most of these occurring only in a few channels.

\section{Discussion}

DOT has an undisputed potential for investigating whole-brain hemodynamics in the neonatal population. ${ }^{6,13,25-31}$ Prior studies in this field have raised the issue related to the cap tolerance by the neonates as a possible factor limiting the duration of DOT data acquisition. Singh et al. ${ }^{6}$ and Chalia et al. ${ }^{13}$ adopted a soft and flexible cap (EasyCap, Germany, i.e., our outer layer), with a whole-head coverage for a multimodal DOT-EEG acquisition. Ferradal and colleagues ${ }^{14}$ employed a custom-made optode holder, composed of five triangular patches joined together with a neoprene headband, fixed to the head via Velcro straps. A layer of flexible plastic was the structural support of the cap and a layer of soft silicone was used to embed the optical fibers. The optode array covered the occipital, temporal, and inferior parietal cortices. Austin et al. ${ }^{32}$ employed a custom-made helmet with whole-head coverage, made of an outer shell of

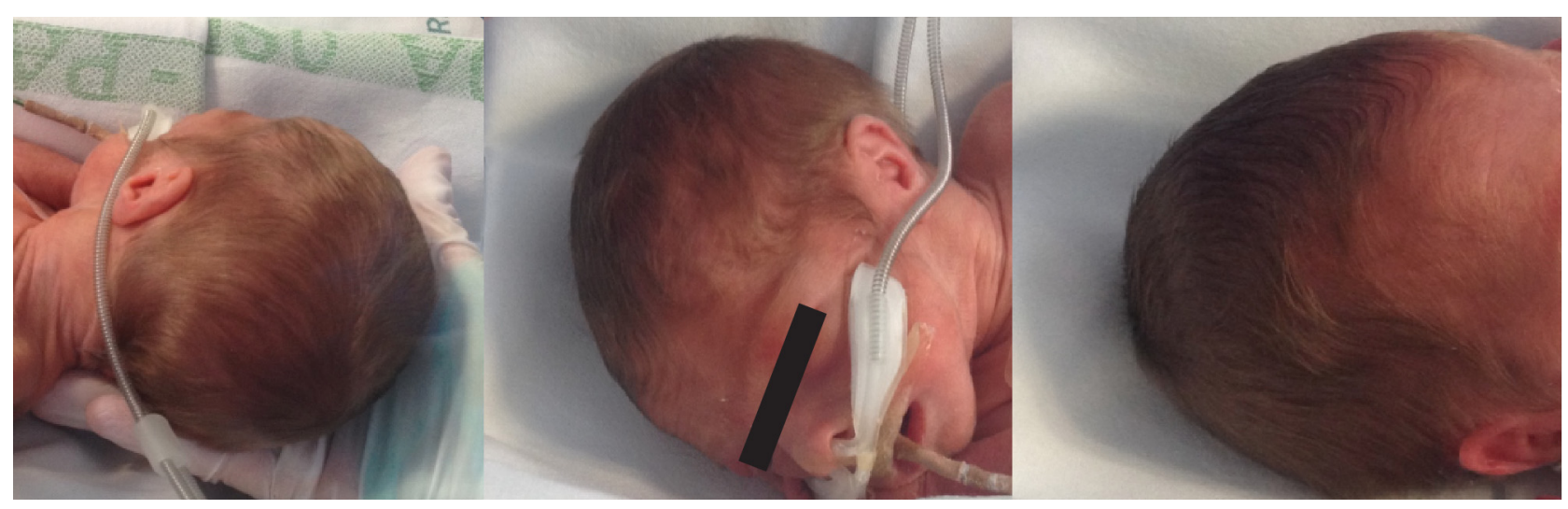

Fig. 2 Pictures of the neonate's head taken after removal of the cap toward the end of the 7-day monitoring period. Neither skin lesions nor evident pressure marks due to the cap can be observed. 

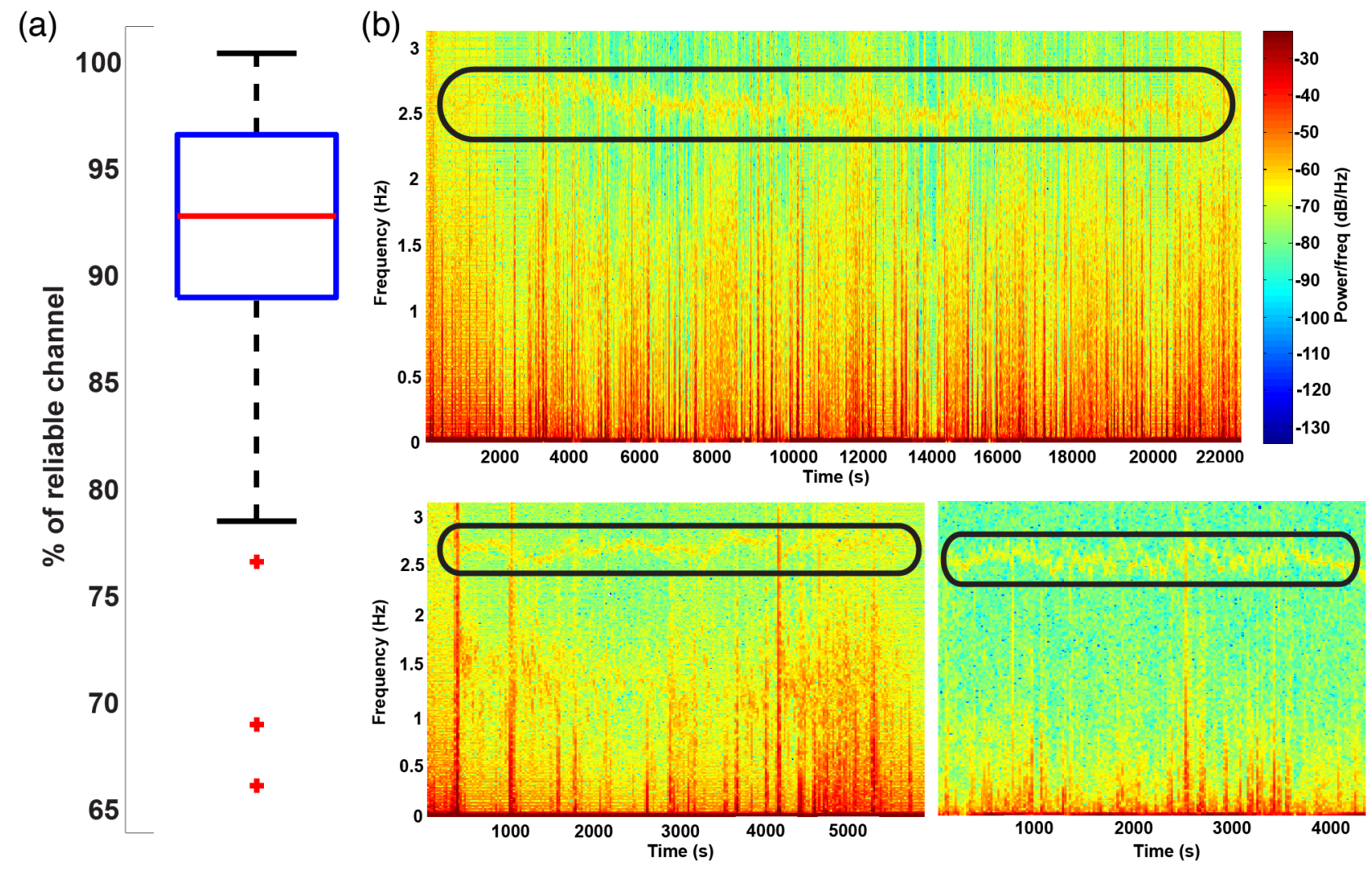

Fig. 3 (a) Box-plot showing the \% of reliable channels during all data segments. The median number of reliable channels across data segment was $92.38 \%$, with only three segments of data having $<78.1 \%$ of reliable channels. (b) Three representative spectrograms, showing the results of the time-frequency analysis. In the upper panel, the spectrogram depicts one segment of data $(\sim 377 \mathrm{~min}$, i.e., $\sim 6 \mathrm{~h}$ and a half) acquired during the first days, in the bottom panel on the left, the spectrogram depicts one segment of data $(\sim 98 \mathrm{~min})$ acquired halfway through the recording phase, and in the bottom panel on the right, the spectrogram depicts one segment of data $(\sim 73 \mathrm{~min})$ acquired toward the end of the 7-day period. The black ellipses highlight in each spectrogram the heartbeat component, which is consistently observable for the entire duration of the three time-series.

thermoplastic and an inner part of NIR-absorbing foam. Crucially, in all these DOT studies, the length of registration did not exceeded $2 \mathrm{~h}$, with a minimal length of $60 \mathrm{~min}^{6,14}$ up to a maximum of $115 \mathrm{~min}^{13}$ and the gestational age ranged from 34 weeks gestation ${ }^{13}$ to term.

Here, we showed that the adoption of our hand-made cap (NeoCAP) enabled a safe continuous DOT monitoring for a 7 -day period in a very preterm infant. Of import, DOT data quality was remarkable, as indicated by the three metrics used to assess the reliability of the measurements; the vast majority of the channels exhibited a high SNR with no noticeable drift, and the heartbeat was easily identifiable in most of the high SNR channels. A (reassuringly) small number of artifacts were identified while the neonate was in quiet sleep, according to EEG traces. Given, however, that identifying quiet sleep in very preterm neonates from an EEG trace is particularly challenging, ${ }^{24}$ our analysis likely overestimated such artifacts, because the probability that the baby was likely to be awake was not negligible. Future studies should consider direct monitoring of sleep stages while acquiring DOT/EEG data.

To date, this represents the longest DOT monitoring ever performed on newborns, and we deem NeoCAP as the critical factor to achieve such a breakthrough, both in terms of monitoring time and signal quality. One of its points of strength is constituted by the presence of a soft inner layer that prevents direct contact between optodes' support/electrodes' support and the neonatal skin, thus avoiding pressure lesions and skin damages. The presence of a spongy inner layer allows postural changes of the newborn without determining increased pressure on a single side due to optodes' support. The current proof-of-concept NeoCAP demonstrates that the appropriate cap may substantially increase the time of continuous DOT monitoring of whole-brain hemodynamics in very preterm neonates. Furthermore, NeoCap can be easily and quickly fitted on and removed from the infant's head as the DOT device could be quickly and easily switched on and off. Nurses could carry on their routine neonatal care with minimal effort required to take care of the cap and the DOT device. The data quality results suggest that nurses were able to successfully fit the cap back on the infant's head after each removal. Furthermore, the use of EasyCap as an external layer had the great advantage to help nurses reliably (re-)position the cap on the newborn's head and reduce the likelihood of misplacement errors. Common landmarks that were used to check the position of the cap on the head were the location of the EasyCap borders, of the holes for the ears and the strip under the chin. The study's physician and the nurse in charge of the newborn checked the positioning of the cap on the newborn's head at least four times per 


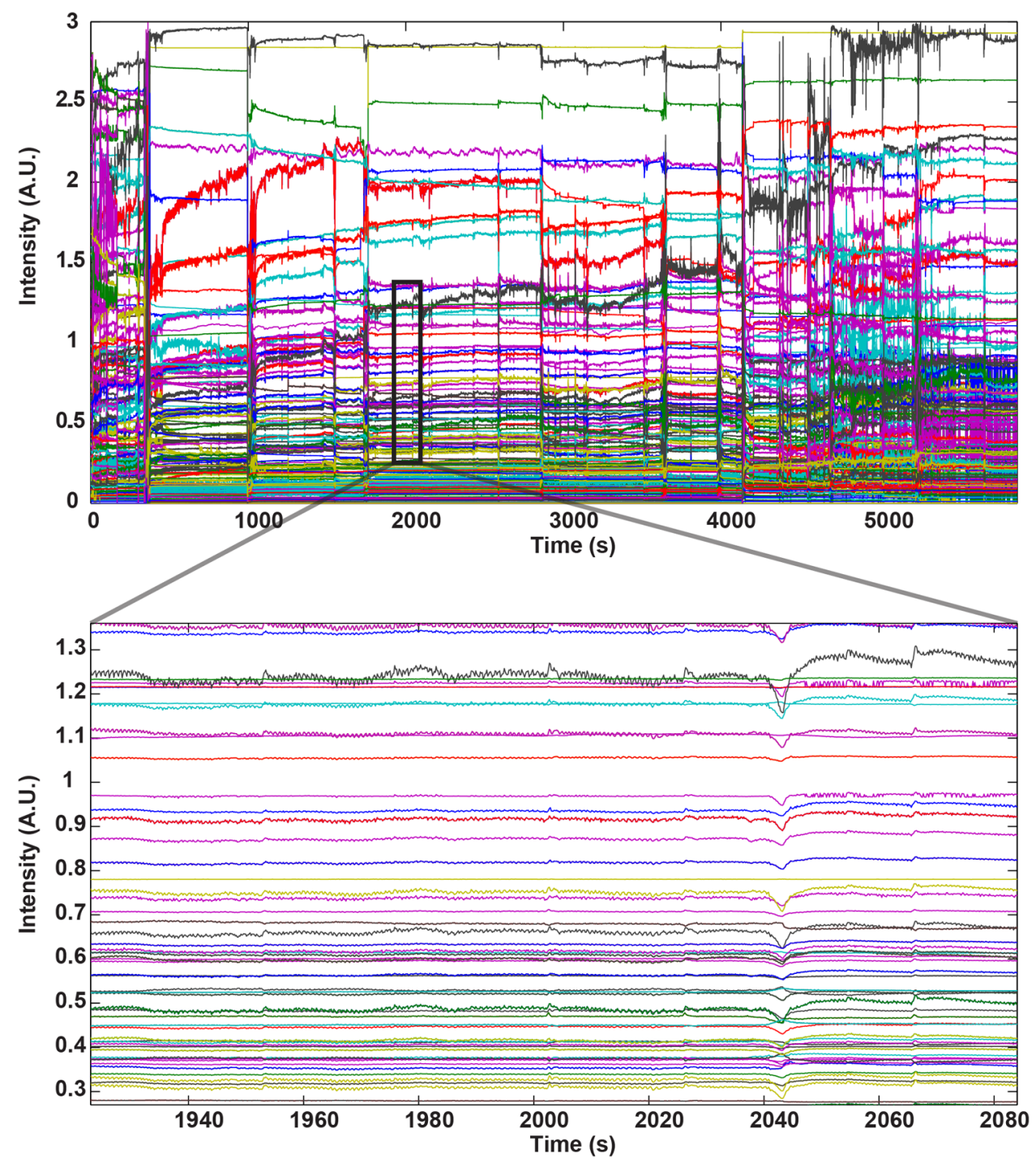

Fig. 4 Example of DOT intensity data of all channels at both wavelengths (210 channels) in one of the data segment. No filtering was applied to the plotted data. In the bottom panel, a particular of the above time-series is displayed. The heartbeat component is appreciable in most of the channels.

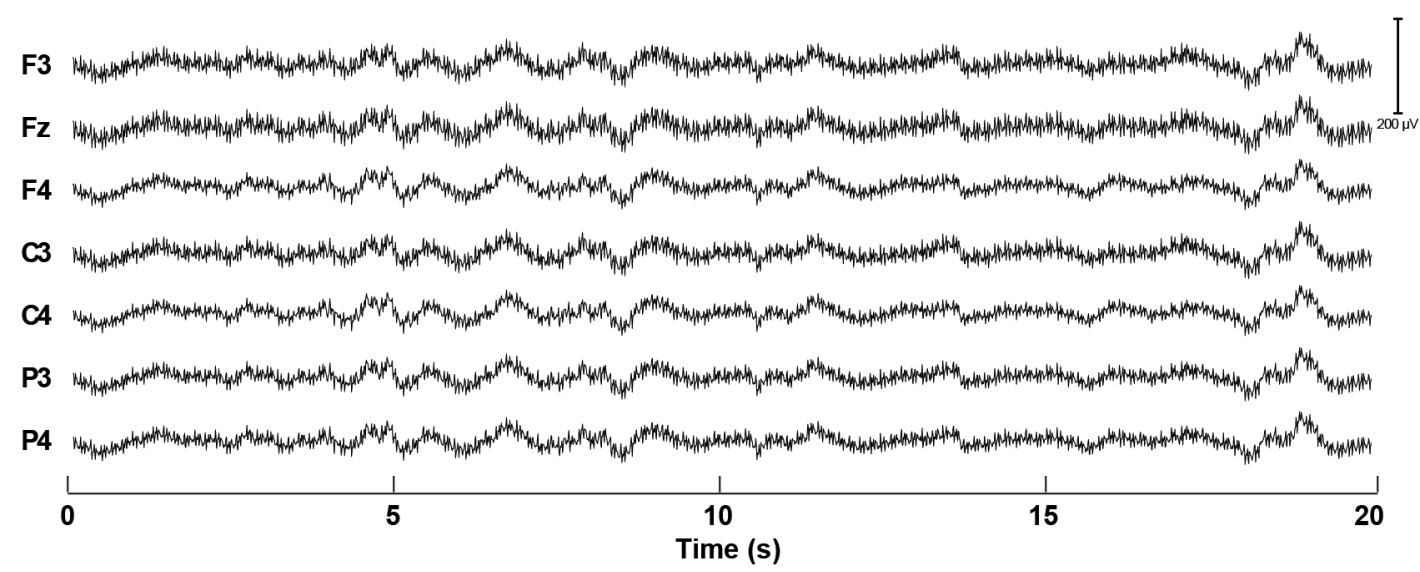

Fig. 5 Example of EEG data. The data were offline re-referenced to the average of FT9 and FT10. A notch filter (cut-off frequency $=50 \mathrm{~Hz}$ ) was applied to remove the line noise. A band-pass filter (cut-off frequency $=0.5$ and $60 \mathrm{~Hz}$ ) was further applied to remove higher frequency noise and low frequency drifts. 
day, and never noticed a misplacement of the cap. Taking pictures of the cap after each repositioning may be a viable solution to quantitatively assess the potential misplacement error.

NeoCAP permits the simultaneous recording of EEG signals when required (e.g., for functional stimulation or for clinical practice). In comparison to DOT, the EEG set up takes longer and electrodes are less comfortable for the infant's skin, due to the presence of the electrolyte gel. The use of cup-like electrodes, filled with the electrolyte gel, could be more appropriate for future studies, because they might reduce the risk of spreading the gel over the inner surface of the cap. Another option would be to embed the electrodes in the inner layer, inside the sponge, so that less electrolyte gel would be needed to lower the impedance.

The low-cost manufacturing process of this head-cap can be replicated to create head-caps for different head sizes, spanning across preterm and term age ranges. Furthermore, optodes' positions and electrodes' positions can be modified during the manufacturing process according to the needs of the study and the number of sources and detectors available. Future studies should attempt to further reduce motion artifacts, which are quite common during the acquisition (although this comes to us as no surprise, given that the cap was not removed during parental visits or other clinical interventions to the baby).

Our work provides evidence that a prolonged continuous DOT monitoring is feasible in preterm infants, highlighting the crucial need for a cap adapted to this specific population to avoid some of the pitfalls in this research field. Adopting NeoCAP might open a new range of exciting opportunities for comparing whole-brain hemodynamic changes with other vital parameters and it might enable to consistently observe the impact of multiple daily stressful events on the brain of preterm infants.

\section{Disclosures}

There is no conflict of interest.

\section{Acknowledgments}

This work was supported by Grant No. STPD11B8HM from the University of Padova. The authors would like to thank the NICU staff for the support and for facilitating the project.

\section{References}

1. F. F. Jöbsis, "Noninvasive, infrared monitoring of cerebral and myocardial oxygen sufficiency and circulatory parameters," Science 198, 1264 1267 (1977).

2. A. T. Eggebrecht et al., "Mapping distributed brain function and networks with diffuse optical tomography," Nat. Photonics 8, 448-454 (2014).

3. S. R. Arridge, "Methods in diffuse optical imaging," Philos. Trans. A. Math. Phys. Eng. Sci. 369, 4558-4576 (2011).

4. S. Brigadoi et al., "Evaluating real-time image reconstruction in diffuse optical tomography using physiologically realistic test data," Biomed. Opt. Express 6, 4719 (2015).

5. S. M. Liao and J. P. Culver, "Near infrared optical technologies to illuminate the status of the neonatal brain," Curr. Pediatr. Rev. 10, 73-86 (2014).

6. H. Singh et al., "Mapping cortical haemodynamics during neonatal seizures using diffuse optical tomography: a case study," NeuroImage. Clin. 5, 256-265 (2014).

7. S. Bembich et al., "The cortical response to a noxious procedure changes over time in preterm infants," Pain 157, 1979-1987 (2016).
8. D. A. Boas et al., "The accuracy of near infrared spectroscopy and imaging during focal changes in cerebral hemodynamics," Neuroimage 13, 76-90 (2001).

9. S. R. Hintz et al., "Bedside functional imaging of the premature infant brain during passive motor activation," J. Perinat. Med. 29, 335-343 (2001).

10. Y. Fuchino et al., "Effects of preterm birth on intrinsic fluctuations in neonatal cerebral activity examined using optical imaging," PLoS One 8, e67432 (2013).

11. B. R. White et al., "Bedside optical imaging of occipital restingstate functional connectivity in neonates," Neuroimage 59, 2529-2538 (2012).

12. S. Cutini and S. Brigadoi, "Unleashing the future potential of functional near-infrared spectroscopy in brain sciences," J. Neurosci. Methods 232, 152-156 (2014).

13. M. Chalia et al., "Hemodynamic response to burst-suppressed and discontinuous electroencephalography activity in infants with hypoxic ischemic encephalopathy," Neurophotonics 3, 031408 (2016).

14. S. L. Ferradal et al., "Functional imaging of the developing brain at the bedside using diffuse optical tomography," Cereb. Cortex 26, 1558-1568 (2015).

15. R. J. Cooper et al., "Transient haemodynamic events in neurologically compromised infants: a simultaneous EEG and diffuse optical imaging study," Neuroimage 55, 1610-1616 (2011).

16. R. Salonia et al., "The utility of near infrared spectroscopy in detecting intracranial hemorrhage in children," J. Neurotrauma 29, 1047-1053 (2012).

17. M. J. Ness, D. M. R. Davis, and W. A. Carey, "Neonatal skin care: a concise review," Int. J. Dermatol. 52, 14-22 (2013).

18. H. J. Vernon et al., "Semipermeable dressing and transepidermal water loss in premature infants," Pediatrics 86, 357-362 (1990).

19. A. Knauth et al., "Semipermeable polyurethane membrane as an artificial skin for the premature neonate," Pediatrics 83, 945-950 (1989).

20. R. Oostenveld and P. Praamstra, "The five percent electrode system for high-resolution EEG and ERP measurements," Clin. Neurophysiol. 112, 713-719 (2001).

21. B. R. N. Stevens et al., "Premature infant pain profile: development and initial valid," Clin. J. Pain 12, 13-22 (1996).

22. T. J. Huppert et al., "HomER: a review of time-series analysis methods for near-infrared spectroscopy of the brain," Appl. Opt. 48, D280-D298 (2009).

23. S. Vanhatalo and K. Kaila, "Development of neonatal EEG activity: from phenomenology to physiology," Semin. Fetal Neonatal. Med. 11, 471-478 (2006).

24. T. Tsuchida et al., "ACNS standardized EEG terminology and categorization for the description of continuous EEG monitoring in neonates," J. Clin. (2013) https://www.acns.org/pdf/guidelines/Guideline-16.pdf (20 November 2016).

25. G. Taga et al., "Spontaneous oscillation of oxy- and deoxy-hemoglobin changes with a phase difference throughout the occipital cortex of newborn infants observed using non-invasive optical topography," Neurosci. Lett. 282, 101-104 (2000).

26. G. Taga et al., "Hemodynamic responses to visual stimulation in occipital and frontal cortex of newborn infants: a near-infrared optical topography study," Early Hum. Dev. 75, 203-210 (2003).

27. M. A. Franceschini et al., "Assessment of infant brain development with frequency-domain near-infrared spectroscopy," Pediatr. Res. 61, 546-551 (2007).

28. N. Roche-Labarbe et al., "Near-infrared spectroscopy assessment of cerebral oxygen metabolism in the developing premature brain," J. Cereb. Blood Flow Metab. 32, 481-488 (2012).

29. N. Roche-Labarbe et al., "Noninvasive optical measures of CBV, StO2, $\mathrm{CBF}$ index, and $\mathrm{rCMRO}_{2}$ in human premature neonates' brains in the first six weeks of life," Hum. Brain Mapp. 31, 341-352 (2010).

30. F. Homae et al., "Development of global cortical networks in early infancy," J. Neurosci. 30, 4877-4882, (2010).

31. M. Imai et al., "Functional connectivity of the cortex of term and preterm infants and infants with Down's syndrome," Neuroimage 85(Pt 1), 272-278 (2014).

32. T. Austin et al., "Three dimensional optical imaging of blood volume and oxygenation in the neonatal brain," Neuroimage 31, 1426-1433 (2006). 
Alfonso Galderisi graduated in medicine at University of Naples and started the residency program in paediatrics at University of Padova, Italy, in 2012. His research interests have involved paediatric diabetes and the application of continuous glucose monitoring in neonatal intensive care. He was recipient of two research fellowships from European Association for Diabetes (EASD, 2014) and from International Society for Paediatric/Adolescent Diabetes (ISPAD, 2016). Currently he is research associate in pediatric endocrinology at University of Yale, New Haven, USA.

Sabrina Brigadoi studied bioengineering before receiving a $\mathrm{PhD}$ in cognitive science from University of Padova, Italy. Formerly a research associate in the Biomedical Optics Research Laboratory at University College London, U.K., she has returned to University of Padova as a postdoctoral fellow. Her research interests are focused on advancing the applicability of diffuse optical techniques in both infant and adult population, with particular interest in signal processing techniques, image reconstruction, and head model development.

Simone Cutini studied psychology before receiving a $\mathrm{PhD}$ in behavioral neuroscience from University of Padova, Italy. He is an assistant professor at the Department of Developmental Psychology, University of Padova. His main research interest is focused on cognitive neuroscience, particularly in exploring the neural substrates of cognitive control, visuo-spatial attention, numerical cognition and visual short-term memory. His line of investigation mainly (although not exclusively) resorts to the use of functional near-infrared spectroscopy (fNIRS).

Sara Basso Moro studied experimental psychology and cognitive sciences at the University of Padova before receiving a $\mathrm{PhD}$ in ultrastructural and molecular imaging from the University of L'Aquila, Italy. She is now working at the University of Padova as a postdoctoral fellow. Her current research interests embrace cognitive neuroscience, in particular visuo-spatial attention and numerical cognition, functional near infrared spectroscopy (fNIRS) and high-density electroencephalography (HD-EEG) in both 5- to 7-month-old newborns and adults.

Elisabetta Lolli is the head nurse of the Neonatal Intensive Care Unit of University of Padova, Italy. She has longstanding experience as an $\mathrm{RN}$ in intensive care unit and heart-lung transplant unit development at the University Hospital of Padova.

Federica Meconi studied experimental psychology before receiving her PhD in cognitive science from University of Padova, Italy. She is currently a postdoctoral fellow at University of Padova and tutor in general psychology course for undergraduate students. Her research interests are focused on event-related potential and oscillatory correlates of human cognition in normative and clinical populations, with particular interests in social cognition and memory processes. She's recently been awarded with a Marie-Skłodowska Curie Actions grant.
Silvia Benavides-Varela studied biotechnology engineering at University of Costa Rica before receiving a $\mathrm{PhD}$ in neuroscience from the International School for Advanced Studies, Italy. Formerly, she was a postdoctoral fellow at the UMR 8242 CNRS-Universitè Paris Descartes, France, and at San Camillo Hospital in Venice, Italy. Currently, she works as a postdoc at University of Padova. Her research interests are mainly focused on infant cognition, in particular language acquisition, and the first stages of memory.

Eugenio Baraldi, MD, FCCP, professor of pediatrics at the Department of Women's and Children's Health of University of Padova, Italy. $\mathrm{He}$ is a head of neonatology and neonatal intensive care, and posthead of the Allergy and Respiratory Diseases Unit. He has more than 150 scientific publications in peer-review journals (including NEJM, Lancet and JAMA). He is a reviewer of research programs for Netherlands Asthma Foundation and The Asthma Foundation of Western Australia. His primary research interests are bronchopulmonary dysplasia, asthma, markers of airway inflammation, and metabolomics.

Piero Amodio, MD, is an associate professor of internal medicine at the University of Padova, Italy. He organized a laboratory and an outpatient clinic for neuropsychological and neurophysiological investigation of metabolic encephalopathies. He coordinates a team and an interdepartmental center for the study of mental changes in internal medicine. He wrote about 160 papers and has an $h$-index $=26$.

Claudio Cobelli is professor of biomedical engineering at University of Padova. His research is in modeling and control of biological systems, supported by NIH, JDRF, and EU. He published 486 papers in refereed journals, is coauthor of 8 books and holds 11 patents with an $h$-index of 88 . He is an associate editor of IEEE Transaction on Biomedical Engineering and Journal of Diabetes Science and Technology. In 2010, he received the Diabetes Technology Artificial Pancreas Research Award. He is fellow of IEEE and BMES.

Daniele Trevisanuto is a consultant neonatologist at the Department of Women's and Children's Health of University of Padova, Italy. He is a member of the International Liaison Committee on Resuscitation, Neonatal Resuscitation task force and a member of the European Resuscitation Council, Neonatal Resuscitation task force. His fields of interest are neonatal resuscitation, education, neonatal airway management, and maternal and neonatal care in low-resource countries. $\mathrm{He}$ is author or coauthor of about 200 peer-reviewed papers.

Roberto Dell'Acqua received a $\mathrm{PhD}$ in cognitive neuroscience in 1998 from University of Padova, Italy. He spent 3 years as a research associate in the labs of Pierre Jolicoeur at the University of Waterloo, Canada, and of Jonathan Grainger at the CNRS Marseille, France. He was appointed full professor of cognitive psychology at the University of Padova in 2001. He is member of the advisory board of the Attention and Performance Society. 